\title{
El cambio pedagógico en la docencia universitaria en los tiempos de Covid-19
}

\section{Pedagogical change in university teaching undergoing Covid-19}

\author{
Marcelo Dorfsman \\ Universidad Hebrea de Jerusalén. Jerusalén, Israel \\ marcelo.dorfsman@mail.huji.ac.il \\ Gabriel Horenczyk \\ Universidad Hebrea de Jerusalén. Jerusalén, Israel \\ gabriel.horenczyk@mail.huji.ac.il
}

\begin{abstract}
Resumen
Antecedentes: La aparición del virus COVID-19 ha generado una de las mayores disrupciones mundiales de los sistemas educativos en generaciones Propósito: El propósito de este estudio es comprender el impacto de una situación de emergencia educativa sobre las prácticas pedagógicas de los docentes universitarios, analizando los focos de resistencia existentes en la implementación de entornos digitales para la enseñanza. Metodología / Enfoque: La investigación siguió un enfoque de métodos mixtos, combinando componentes cualitativos con cuantitativos. El presente estudio pone especial énfasis en los componentes cualitativos de la investigación. Resultados / Conclusiones: El estudio encontró que existen dos dimensiones en la disposición al cambio pedagógico genuino en los docentes universitarios: Una es su nivel de alfabetización digital antes de la crisis; la segunda es su concepción pedagógica de enseñanza. En el trabajo, se analiza el modelo de aceptación de tecnologías desarrollado por Venkatesh y colaboradores (Modelo UTAUT), y se lo enriquece con una tipología de aceptación del cambio pedagógico (TACP) que permite comprender la situación planteada y actuar en consecuencia. Implicaciones: Este modelo se puede aplicar a diversas situaciones de crisis y puede ser útil para la formación y desarrollo del profesorado universitario en el campo de la alfabetización digital tomando en cuenta las limitaciones que se indican al final del trabajo.

Palabras clave: Enseñanza en línea, Enseñanza remota de emergencia, Docencia universitaria, Alfabetización digital.
\end{abstract}

\begin{abstract}
Background: The emergence of the COVID-19 virus has generated one of the largest global disruptions of education systems. Purpose: The purpose of this study is to better understand the impact of educational emergency situation on the pedagogical practices of university teachers, analyzing the areas of resistance to the implementation of digital environments to teaching. Methodology / Approach: The research followed a mixed methods approach, combining qualitative and quantitative components. It places special emphasis on the qualitative components of the research. Results / Conclusions: The study identified two dimensions in the disposition to genuine pedagogical change in university teachers: One is their level of digital literacy before the crisis; the second is his pedagogical conception of teaching. In this work the model of acceptance of technologies developed by Venkatesh and colleagues (UTAUT Model) is analyzed, and is enriched with a typology of acceptance of pedagogical change (TACP) that allows the researchers to understand the situation and act accordingly. Implications: This model can be
\end{abstract}


applied to different crisis situations and can be useful for the training and development of university teachers in the field of digital literacy, taking into account the limitations indicated at the end of the work.

Keywords: Online Teaching, Emergency Remote Teaching, University Teaching, Digital Literacy.

\section{Introducción}

Este estudio se realizó en los días de extrema crisis del Covid 19 en Israel, en el marco de una pandemia que ya se dimensionaba a nivel mundial. El 10.3.2020, la administración de la Universidad Hebrea anunció que la apertura del semestre, que estaba prevista para el 15.3.2020, se tendría que posponer una semana, y se llevaría a cabo principalmente a través de la enseñanza en línea. La semana previa al inicio del semestre sería utilizada para la preparación frente a la nueva situación de emergencia educativa.

En esas circunstancias, la Unidad de Educación a Distancia de la Universidad puso a disposición de los docentes una serie de instructivos y capacitación intensiva a principalmente través de Zoom. La universidad ya había adquirido licencias de Zoom y de Panopto para todos los docentes.

Las clases comenzaron el 22.3.2020, con aproximadamente 25,000 estudiantes y unos 2500 docentes, a través de dichas plataformas.

La enseñanza mediada por entornos digitales existe ya hace más de dos décadas; y a lo largo de las mismas, se ha desarrollado de manera lenta, progresiva y desigual en diferentes países e instituciones. Al iniciar el semestre, los docentes de la Universidad Hebrea, se vieron "forzados", como tantos otros, a enseñar en un entorno digital que para la mayoría era nuevo y en ocasiones, no confiable para la enseñanza.

Este trabajo se focaliza en dos ejes principales: por un lado, los investigadores se preguntan acerca de los modos de utilización de los entornos digitales en una situación de emergencia educativa y en la posibilidad de que éstos puedan o no generar cambios profundos y duraderos en la enseñanza; por el otro, el trabajo hace foco en los modos de aceptación de los docentes de la tecnología digital en sus prácticas de enseñanza. Un aspecto relevante a tomar en cuenta es la dimensión institucional que, si bien no es su foco principal, es parte inherente del mismo.

El objeto de análisis en este trabajo es la práctica pedagógica de los docentes en situaciones de emergencia, y la factibilidad del cambio pedagógico en estas circunstancias. Un componente central en dicho análisis es el grado de aceptación del uso de tecnologías considerado como una de las condiciones del mismo.

La pregunta central es entonces si efectivamente, una situación forzada puede llevar a cambios profundos, y cuáles son, en este caso, los factores favorecedores y obstaculizadores de los mismos.

Los objetivos de la investigación son:

- Comprender el impacto de una situación de emergencia educativa sobre las prácticas de enseñanza de los docentes universitarios.

- Desarrollar una tipología docente que dé cuenta de las diferentes modalidades de reacción ante situaciones de emergencia.

Dorfsman, M. \& Horenczyk, G. El cambio pedagógico en la docencia universitaria en los tiempos de Covid-19 
- Analizar los focos de resistencia de los docentes en la implementación de entornos digitales a sus prácticas de enseñanza.

- Comprender la relación, si es que la hay, entre el grado de alfabetización digital de los docentes y su disposición a emprender cambios profundos en sus prácticas pedagógicas.

De estos objetivos se han derivado las siguientes preguntas de la investigación:

- ¿En qué medida una situación de crisis puede impactar en la disposición del docente universitario a efectuar cambios pedagógicos en su práctica?

- ¿Qué tipos de cambios pedagógicos se produjeron, si es que los hubo, en la práctica pedagógica de los docentes universitarios en tiempos de la enseñanza bajo el impacto de la Crisis de Enseñanza Relacionada con el Corona (CRTS) ${ }^{1}$ ?

- ¿Cuáles son los factores favorecedores y obstaculizadores de dichos cambios?

Los investigadores consideran que una comprensión profunda de los procesos que se desencadenan en situaciones de emergencia educativa - centrados principalmente en la práctica docente y en la dimensión institucional- contribuirá al mejoramiento de procesos similares en otras instituciones educativas tomando en cuenta las limitaciones que se señalarán al final del trabajo.

\section{Marco Teórico}

El surgimiento de COVID-19 posiblemente haya generado la mayor disrupción de sistema educativo que hemos presenciado en la era moderna. En palabras de Audrey Azoulay, director de la UNESCO "Nunca antes habíamos presenciado una interrupción educativa a tan gran escala ...". Según la UNESCO, el $87 \%$ de la población estudiantil mundial, en 165 países, ha sufrido el cierre de sus instituciones (UNESCO, 2020).

Estudios realizados en situaciones de emergencia ocurridas en el pasado, como la epidemia de $\mathrm{SARS}^{2}$, pueden servir de antecedente de la situación actual, aunque los volúmenes son sustancialmente diferentes. En este sentido, Hodges y otros diferencian entre una enseñanza en línea planificada y eficaz, y lo que ellos llaman Emergency Remote Teaching (ERT) (Hodges, Moore, Lockee, Trust, \& Bond, 2020). La diferencia entre los dos es que el segundo ofrece una modalidad de enseñanza temporal, que le permite continuar mientras dure la crisis, pero no pretende introducir cambios profundos en el sistema educativo para hacerlo más efectivo y eficiente. La implementación de este tipo de enseñanza está marcada por un alto grado de enseñanza asincrónica, que puede garantizar un aprendizaje intensivo y resultados razonables. Si bien Hodges y otros (2020) consideran una clara diferenciación entre el ERT y la enseñanza en línea, Murphy (2020) establece la necesidad de "normalizar una pedagogía pospandémica, en la que la enseñanza en línea forma parte de una nueva normalización del e-Learning de emergencia, que se refiere a estrategias que enmarcan la adopción generalizada del aprendizaje en línea bajo COVID-19 como un camino hacia una nueva respuesta normal en lugar de una respuesta de emergencia"(Murphy, 2020) p. 10).

\footnotetext{
${ }^{1}$ CRTS: Corona Related Teaching Situation, del original en inglés, denominación asumida por el equipo internacional de investigación.

2 Dicha epidemia, que acaeció en el año 2003, afectó a diferentes ciudades de China y Extremo Oriente, y entre ellas Hong Kong.
}

Dorfsman, M. \& Horenczyk, G. El cambio pedagógico en la docencia universitaria en los tiempos de Covid-19 
Entonces es dable considerar, como Yan (2020), dos posibles escenarios para caracterizar el actual período de pandemia: por un lado, puede convertirse en una gran oportunidad para generar cambios profundos en la docencia universitaria, ayudados por el uso de herramientas y entornos digitales (Yan, 2020); por el otro, una percepción más conservadora y pesimista sugiere que después del período de ERT, el retorno a una supuesta normalidad no garantiza cambios profundos y duraderos (Hodges et al., 2020).

¿A qué se denomina "cambios profundos" en la práctica pedagógica? Tarling y Ng'ambi (2016) proponen un esquema teórico según el cual el cambio en las prácticas pedagógicas ocurre en la transición de lo que ellos denominan "pedagogías de transmisión" a las "pedagogías de transformación". En las primeras se priorizan las actividades cognitivas más bajas en la escala de Bloom, como comprender, memorizar, recordar; y en las segundas se priorizan las actividades cognitivas superiores, tales como analizar, comparar, crear y evaluar (Tarling \& Ng'ambi, 2016).

Diversos investigadores han destacado el potencial de las TIC para promover prácticas más centradas en el alumno que favorezcan su actividad, creatividad, trabajo en equipo y con los compañeros (Beetham \& Sharpe, 2007; McLoughlin \& Lee, 2008). Las teorías del cambio pedagógico destacan diferentes tipos de resistencia de los docentes al cambio, desde la noción de "riesgo" por el cambio, pasando por la falta de conocimiento, apoyo institucional, visualización del beneficio del cambio, entre otros (Le Fevre, 2014; Tarling \& Ng'ambi, 2016; Terhart, 2013).

Asimismo, se ha investigado en profundidad la disposición de los sujetos en general, y de los docentes en particular, para utilizar tecnologías en sus prácticas, desarrollándose diferentes modelos que tratan de explicar los procesos de aceptación de las tecnologías. Fred Davis, en su Modelo de aceptación de tecnologías, $\left(\mathrm{TAM}^{3}\right)$ sostiene la existencia de dos factores determinantes: La percepción de utilidad y la percepción de facilidad de uso (Davis, 1989). Este modelo fue expandido sucesivamente a los modelos TAM2 y TAM3 (Venkatesh \& Davis, 2000) incluyendo aspectos que tienen que ver con la influencia social y la auto-eficacia.

Por último, Admiraal y otros adoptaron la Teoría unificada de aceptación y uso de tecnología, de Venkatesh, (UTAUT ${ }^{4}$ ),(Admiraal et al., 2017; Venkatesh \& Bala, 2008; Venkatesh, Morris, Davis, \& Davis, 2003). Este modelo intenta unificar los diferentes modelos existentes y se basa en cuatro categorías que Venkatesh y sus colegas consideran como determinantes para el uso de tecnologías (Venkatesh et al., 2003).

La primera es la "expectativa de desempeño" que se define como "el grado en que un individuo cree que el uso del sistema lo ayudará a obtener ventajas en su desempeño laboral" (Venkatesh et al., 2003, p. 447). En los diferentes modelos analizados por los autores, este parece ser el predictor más significativo del uso de tecnologías en términos de motivación, percepción de éxito y contexto social.

La segunda es la "expectativa de esfuerzo", definida como "el grado de facilidad asociado con el uso del sistema" (Venkatesh et al., 2003, p. 450). Esta categoría es de especial relevancia para los primeros periodos de uso de tecnologías, y se refiere a la

\footnotetext{
${ }^{3}$ Las siglas corresponden al original en inglés: Technology Acceptance Model (MD-GH).

${ }^{4}$ Las siglas corresponden al original en inglés: Unified Theory of Acceptance and Use of Technology (MD$\mathrm{GH})$.
}

Dorfsman, M. \& Horenczyk, G. El cambio pedagógico en la docencia universitaria en los tiempos de Covid-19 
percepción de la complejidad de la tarea a abordar y el esfuerzo requerido para llevarla a cabo.

La tercera categoría es la "influencia social" definida como "el grado en que un individuo percibe que otras personas importantes creen que debería usar el nuevo sistema" (Venkatesh et al., 2003, p. 451). Esta categoría se refiere a la percepción de la aceptación de tecnologías por parte de figuras relevantes en su contexto sociolaboral, así como la relevancia de ésta para la construcción de su propia imagen.

La cuarta y última categoría refiere a las "condiciones facilitadoras", que se definen "como el grado en que un individuo cree que existe una infraestructura organizativa y técnica para apoyar el uso del sistema" (Venkatesh et al., 2003, p. 453). Se refiere a la medida en que los individuos perciben que podrán controlar los sistemas por sí mismos, con asistencia tecnopedagógica (recursos humanos y materiales) y con el apoyo técnico y administrativo de la institución.

Otro modelo a tomar en cuenta es el modelo integrador de predicción del comportamiento $\left(\mathrm{IMBP}^{5}\right)$ y su adaptación a las prácticas docentes (Fishbein \& Ajzen, 2011; Kreijns, Vermeulen, Kirschner, Buuren, \& Acker, 2013). En el mismo, se ponen de relevancia tres categorías para explicar y predecir las actitudes de los docentes respecto del uso de las tecnologías: Las actitudes, las normas percibidas y la autoeficacia en el marco de las destrezas y las limitaciones del entorno. Las actitudes reflejan la sensación general del docente respecto del uso de las tecnologías; las normas percibidas dan cuenta de la percepción de uso general de las tecnologías en el entorno laboral, y también el uso efectivo de otros docentes. La autoeficacia es percibida como la propia capacidad de los docentes para utilizar TIC. Ambos modelos han sido producto de rigurosas investigaciones de corte cuantitativo que tratan de analizar las circunstancias bajo las cuales los docentes son capaces o bien están dispuestos a utilizar tecnologías.

\section{Metodología}

Esta investigación sigue un enfoque de método mixto, que combina componentes cualitativos con cuantitativos. Según Creswell y Clark (2017), los cuatro tipos principales de diseños de métodos mixtos son el diseño de triangulación, el diseño incrustado, el diseño explicativo y el diseño exploratorio. El método adoptado en este estudio es un diseño explicativo secuencial de métodos mixtos, que consta de dos fases distintas: una fase cuantitativa seguida de otra cualitativa (Creswell \& Clark, 2017).

El Diseño Explicativo (también conocido como Diseño Secuencial Explicativo) es un diseño de enfoque de métodos mixtos de dos fases, que comienza con la recopilación y análisis de datos cuantitativos. A esta fase preliminar le sigue la posterior recopilación y análisis de datos cualitativos. Esta fase cualitativa, que es la principal de este estudio, está diseñada de modo tal que se desprenda de (o se conecte a) los resultados de la fase cuantitativa preliminar.

A continuación, se ofrece una explicación detallada del método mixto y de los métodos de análisis cuantitativo y cualitativo utilizados en esta investigación.

En la fase cuantitativa de la investigación se hizo uso de un cuestionario en línea que constaba de 33 ítems divididos en tres partes, a saber; 1: La enseñanza antes de la CRTS; 2; La enseñanza durante la CRTS; y 3: Enseñar después de la situación de la CRTS.

\footnotetext{
${ }^{5}$ Refiere a las siglas del inglés en su versión original: Integrative Model of Behaviour Prediction (MD-GH)
}

Dorfsman, M. \& Horenczyk, G. El cambio pedagógico en la docencia universitaria en los tiempos de Covid-19 
El propósito del cuestionario era obtener un primer nivel de información de una muestra representativa del colectivo docente de la Universidad Hebrea; la información obtenida en el cuestionario se ampliaría y profundizaría en la etapa cualitativa a través de las entrevistas en profundidad semiestructuradas.

En la primera parte del cuestionario se solicitó información general acerca de las prácticas de enseñanza y del uso de tecnologías antes de CRTS. Ejemplos de preguntas incluidas en esta parte: ¿en qué medida usted utilizaba herramientas digitales en la enseñanza antes del CRTS? En su opinión, ¿en qué medida las herramientas digitales enriquecían su enseñanza convencional?

En la segunda parte del cuestionario, se inquirió acerca de la confrontación con la CRTS. Ejemplos de preguntas incluidas en esta segunda parte: De acuerdo con su experiencia docente en general y su experiencia en la enseñanza en línea en particular, ¿cómo describiría su experiencia docente durante CRTS ?, ¿En qué medida cree que el éxito de su enseñanza en línea en el contexto actual se debe a alguno de las siguientes razones?: mis habilidades tecnológicas; mi apertura y-o disposición para aprender nuevos métodos de enseñanza; el apoyo pedagógico-tecnológico de la institución; la inversión de tiempo en la preparación de lecciones.

En la tercera parte se preguntó sobre "el día después". El objetivo en esta sección era comprender si, y en qué medida, el encuestado entendía que la experiencia de la enseñanza en línea bajo CRTS afectaría la práctica pedagógica al regresar a la enseñanza presencial. Ejemplos de preguntas: ¿Cree que su nueva experiencia en el uso de herramientas digitales afectará su práctica pedagógica? ¿En qué medida piensa incorporar lecciones a través del sistema de videoconferencia en los cursos convencionales que impartirá en el futuro?

El desarrollo del cuestionario implicó un proceso de consulta entre los miembros de un equipo internacional con el que se compartió la investigación. Se discutió la redacción de algunos ítems, así como las escalas de respuesta apropiadas para el análisis.

Antes de la administración de los cuestionarios, los investigadores de cada uno de los sitios confirmaron con otros colegas la claridad y coherencia del cuestionario. Tras una última ronda de revisiones, se procedió a la difusión.

La técnica utilizada para la selección de la muestra fue el muestreo probabilístico aleatorio al azar, en la cual cada elemento de población tienen una probabilidad igualmente cuantificable de ser seleccionado (Argibay, 2009).

En una primera etapa, el cuestionario se administró a todo el personal docente de la Universidad y se recibieron 241 respuestas $^{6}: 53 \%$ de los encuestados hombres, y $47 \%$ mujeres; El 27\% informó que tienen de 1 a 5 años de experiencia, el 22\% de 6 a 11 años, el 20\% informó de 12 a 17 años de experiencia y el 32\% de 18 años o más. En cuanto a las áreas de instrucción, el 23\% correspondió a las Ciencias Sociales, el 22\% a las Humanidades, el 8\% a las Ciencias Naturales y el resto se dividió entre otras áreas.

Los datos expuestos dieron la pauta a los investigadores acerca de la representatividad de la muestra, dado que los rasgos de los elementos que la integraban eran similares a los de toda la población y la muestra reproducía de manera aproximada a la totalidad de la población docente universitaria.

\footnotetext{
${ }^{6}$ El total de docentes de la Universidad era de alrededor de 2500 , siendo los que efectivamente estaban en condiciones de dar clases en el momento de suministrar el cuestionario eran entre 1500 y 1800 , cifra a la que se llega restando a los que estaban de licencia, año sabático, aquellos que enseñan solamente durante el primer cuatrimestre, etc, de acuerdo a datos suministrados por la dirección de RRHH de la Universidad.
}

Dorfsman, M. \& Horenczyk, G. El cambio pedagógico en la docencia universitaria en los tiempos de Covid-19 
El análisis cuantitativo del cuestionario consistió en estadísticas descriptivas (principalmente frecuencias) y correlaciones entre variables relevantes seleccionadas por el equipo investigador.

En el cuestionario, se solicitó a los docentes indicar si estarían dispuestos a participar en la etapa de investigación cualitativa mediante la realización de una entrevista en profundidad de aproximadamente una hora, con el fin de ampliar y profundizar los datos solicitados en el mismo. Para definir la muestra entre las 110 respuestas positivas obtenidas, se optó por la técnica del muestreo por juicio; en este tipo de muestreo, de acuerdo con los principios de la representatividad estructural, las variables que delimitan la composición estructural de la muestra se eligen de acuerdo con criterios teóricos previamente acordados. El número de unidades requeridas se estableció de acuerdo con el principio del punto de saturación (Bertaux, 1993; Navarrete, 2000).

Los investigadores eligieron dos variables en la selección de la muestra; los años de experiencia y el campo disciplinar de enseñanza. En la muestra obtenida, 10 docentes pertenecían al género femenino y 5 al masculino; 10 enseñaban en las humanidades y 5 en las ciencias naturales; 6 docentes informaron entre 1 y 5 años de experiencia, 3 entre 6 y 11 años, 2 entre 12 y 17 años y 4 informaron 18 años o más de experiencia docente.

Los 15 docentes seleccionados participaron de una entrevista semiestructurada, que duró aproximadamente una hora. Las entrevistas fueron grabadas y posteriormente transcriptas para su análisis, para el cual se utilizó el método de análisis temático (Braun \& Clarke, 2012).

La cantidad de entrevistas realizadas permitió alcanzar el punto de saturación, es decir, el momento en que los nuevos casos tienden a repetir - saturar- el contenido del conocimiento anterior (Bertaux, 1993; Navarrete, 2000).

El guión de la entrevista se realizó tomando en cuenta básicamente las tres etapas que componían el cuestionario: la enseñanza previa a la CRTS, la confrontación con la situación de emergencia y "el día después".

La entrevista abordó cinco temas: a. Antecedentes docentes, por ejemplo; ¿En qué áreas trabaja? ¿Cuántos años llevas en la (tu) Universidad? ¿Cuántos en la enseñanza?; b. Concepciones de enseñanza; por ejemplo: ¿Cómo suele preparar sus clases? ¿Qué tipo de recursos utiliza? Antes de la crisis, ¿utilizaba herramientas tecnológicas? ¿Cómo? ¿Cuáles?; c. El impacto de la enseñanza en línea en sus prácticas; por ejemplo: ¿Cómo pudo afrontar la CRTS? ¿Ha tenido la posibilidad de prepararse para ella? Además de la plataforma zoom, ¿utilizó otras herramientas? ¿Ha notado diferencias entre la primera y la última clase que ha enseñado? ¿Qué tipo de diferencias?; d. La perspectiva institucional; por ejemplo: ¿Qué rescata y qué podría haber sido mejor en el desempeño institucional? ¿Sintió que podía manejarse libremente en su enseñanza?; e. El día después: ¿Cómo prevé el regreso a las clases convencionales? ¿Cómo concibe la nueva situación? ¿Le gustaría volver al tipo de práctica que desarrollaba antes de la crisis? ¿Cree que es probable que ésta cambie?

El análisis se realizó utilizando la metodología del análisis temático (Braun \& Clarke, 2012). Esta metodología posibilita identificar, organizar y ofrecer información fehaciente sobre patrones de significado (temas) en un conjunto de datos de manera sistemática. La principal ventaja de la misma es su flexibilidad para acceder a los datos y para encarar su análisis. En el marco de esta metodología, se implementó un enfoque deductivo "en el

Dorfsman, M. \& Horenczyk, G. El cambio pedagógico en la docencia universitaria en los tiempos de Covid-19 
cual el investigador aporta a los datos una serie de conceptos, ideas o temas que utiliza para codificar e interpretar los datos" (Braun \& Clarke, pág. 3). En el mismo prevalece la interpretación semántica; la orientación es crítica y favorece la triangulación con la literatura científica para la construcción teórica.

En una primera etapa del análisis temático-deductivo, se codificaron las entrevistas según ocho ejes que claramente derivan de los temas tratados en las mismas: 1. Concepción pedagógica de los docentes; 2. La inclusión de las TIC en la enseñanza; 3. Buenas prácticas; 4. Confrontación de los docentes con la CRTS; 5. El impacto del CRTS en las prácticas pedagógicas; 6 . El concepto de evaluación; 7. La perspectiva institucional; 8. Regreso al aula.

En primer lugar, los párrafos considerados significativos en cada entrevista se colorearon, de acuerdo al eje temático correspondiente; dicha información se volcó a una plataforma Zotero $^{7}$, que permitió organizar las categorías definidas, incluir en cada categoría los textos significativos de cada entrevista (previamente coloreados), establecer "tags" que permitan realizar búsquedas internas y articulaciones entre categorías, definir palabras clave y convertir la información obtenida en las entrevistas, en material útil para el análisis (Kvale, 1996; Shkedi, 2003).

En una segunda lectura, los autores decidieron unificar categorías de acuerdo con un criterio de interpretación semántica. De este modo la categoría "buenas prácticas" quedó incluida en "inclusión de TIC en la enseñanza"; "el concepto de evaluación" quedó incluido en "la concepción pedagógica de los docentes"; y la categoría "regreso al aula" quedó incluida en "El impacto del CRTS en las prácticas pedagógicas". De este modo, los ejes temáticos se simplificaron a 5 categorías: 1: concepciones pedagógicas de los docentes; 2: Inclusión de las TIC en la enseñanza; 3: Confrontación de los docentes con la CRTS; 4: El impacto de la CRTS en las prácticas pedagógicas; 5. La perspectiva institucional.

El análisis temático se llevó a cabo en base a la interpretación semántica de los fragmentos seleccionados de las entrevistas, acompañado de un análisis en base a palabras claves existentes (Ortner, 1973; Strauss \& Corbin, 2007).

El procedimiento de codificación se llevó a cabo utilizando la herramienta "wordle", con la cual se analizaron las palabras recurrentes en cada entrevista. Así, por ejemplo, para la sub-categoría: "enfoque centrado en el docente", se encontraron las siguientes palabras y expresiones: "(Yo) organizo", "(yo) estimulo", "profesor", "tiempo", "debate"; en tanto para la sub-categoría de "enfoque centrado en el estudiante", se encontraron "reciben", "necesitan", "feedback", "material (de trabajo", "bonus". Adicionalmente, cada una de las sub-categorías desarrolladas se triangulaban con literatura científica existente. De esta manera, se definieron y operacionalizaron las sub-categorías que se desarrollaron durante el análisis temático-deductivo con énfasis en la dimensión semántica y teórica.

En el próximo capítulo se presentarán los tres perfiles docentes obtenidos, producto del análisis llevado a cabo durante el proceso de análisis: los profesores experimentados, los entusiastas y los cautelosos.

\footnotetext{
${ }^{7}$ Se trata de una plataforma útil para realizar diferentes tareas en el marco de una investigación. Para más Información ver https://www.zotero.org/
}

Dorfsman, M. \& Horenczyk, G. El cambio pedagógico en la docencia universitaria en los tiempos de Covid-19 


\section{Resultados de la investigación}

Tal como se indica en el capítulo metodológico, en el proceso de investigación se analizaron en primer lugar los datos obtenidos de los cuestionarios, mediante la realización de estadísticas descriptivas y correlaciones entre variables relevantes seleccionadas por los autores. En una segunda etapa se efectuó el análisis cualitativo.

A los efectos de organizar adecuadamente los datos y de acuerdo al método mixto utilizado, a continuación se presentan los resultados de investigación en base a las categorías derivadas del análisis temático-deductivo. Al explicar cada una, se alternan los datos obtenidos del análisis cuantitativo - cuando es pertinente - y luego, los resultados del análisis cualitativo que profundizan y enriquecen a los primeros.

En una primera etapa, el análisis temático-deductivo utilizado nos permitió identificar perfiles docentes en relación con tres dimensiones de análisis: el cambio pedagógico, el nivel de alfabetización digital, y la concepción pedagógica de los docentes.

Una vez definidos los perfiles, en una segunda etapa se procedió a articularlos con el modelo de aceptación de tecnologías (UTAUT).

\section{Cambio pedagógico, concepción pedagógica y alfabetización digital de los docentes}

Tal como se indica en nuestro capitulo metodológico, el punto de partida es el análisis de las cinco categorías temáticas encontradas:

\section{Concepciones pedagógicas de los profesores}

En esta primera categoría no se incluyen datos cuantitativos. En la misma se agrupan las respuestas de los profesores a "¿cómo se enseña?" que se les presenta en la primera parte de la entrevista y se refiere a la enseñanza previa al CRTS. Cada docente se refiere espontáneamente a los aspectos centrales de su enseñanza: cuáles son sus énfasis, sus inquietudes, sus tareas y también cuál fue el uso que hicieron de las tecnologías antes de la CRTS.

En las respuestas, se identifican inicialmente dos subtemas o enfoques conceptuales bien definidos: el enfoque centrado en el docente $\left(\mathrm{TCA}^{8}\right)$ y el enfoque centrado en el estudiante $\left(\mathrm{SCA}^{9}\right)$ (Admiraal et al., 2017; Brown, 2003; Emaliana, 2017; Kálmán, Tynjälä, \& Skaniakos, 2020). Los investigadores consideraron que estas dos categorías no lograban explicar por completo el material conceptual obtenido, para lo cual se ha agregado un tercer enfoque, que es el enfoque centrado en el contenido $\left(\mathrm{CCA}^{10}\right)$, al cual Dorfsman, Fenstermacher y Soltis también refieren como al enfoque profesional (Dorfsman, 2018; Fenstermacher \& Soltis, 2004).

A continuación, se presenta el detalle de cada uno de estos enfoques conceptuales:

\footnotetext{
${ }^{8}$ Teacher Centered Approach (del original en inglés).

${ }^{9}$ Student Centered Approach (del original en inglés).

${ }^{10}$ Content Centered Approach (del original en inglés).
}

Dorfsman, M. \& Horenczyk, G. El cambio pedagógico en la docencia universitaria en los tiempos de Covid-19 


\section{Docentes orientados al enfoque basado en el docente (TCA):}

Este primer grupo incluye profesores que centran su respuesta en qué pueden "provocar" en los alumnos, qué proponer, qué y de qué forma transmitir su contenido. Algunos están más interesados en la transmisión de contenidos; otros están más ocupados en el cómo; cómo generar debates, de qué manera generar interacción entre los estudiantes y consigo mismos.

“..., creo que tengo una muy buena capacidad analítica, entiendo muy bien los temas y tengo una muy buena capacidad para simplificarlos y decir todo de una manera muy simple y sin complicaciones, y con palabras no demasiado difíciles" (Noa) ${ }^{11}$. "[La clase] no funciona si no llego con algún programa ordenado de enseñanza. Quiero decir, si no tengo en mente el patrón exacto de lo que voy a decir y simplemente empiezo a hablar" (Avigdor).

\section{Docentes orientados al enfoque basado en contenidos (CCA):}

Se ha incluido en este segundo grupo a quienes, al hablar de su enseñanza, hacen mención inmediata a la estructura epistemológica de los contenidos;

"Trabajo con un modelo de pensamiento educativo que dice que toda concepción filosófica se construye desde la concepción de la persona digna, la persona existente y las técnicas educativas" (Moshe). Ellos basan su instrucción en trabajos de exploración, dado que les interesa que sus alumnos puedan investigar;

"Los materiales que enseño pertenecen a la investigación cuantitativa, por lo que generalmente las lecciones son aquellas en las que se hace alguna pregunta" (Michael).

A diferencia del anterior, este grupo de profesores está menos centrado en su práctica y más centrado en el contenido que enseñan y, por lo tanto, en la comprensión de este contenido por parte de los estudiantes.

\section{Docentes orientados al enfoque basado en el estudiante (SCA):}

Este grupo de docentes está especialmente preocupado por la comprensión del alumno, construye la clase teniendo en cuenta el nivel de comprensión de sus alumnos y no se preocupa por invertir tiempo extra en clase, si esto va a contribuir a que todos tengan éxito.

A diferencia del primer grupo, y en menor medida del segundo, estos docentes tienen como objetivo explícito que sus alumnos comprendan y triunfen a pesar de sus dificultades y del esfuerzo extra que esto les pueda suponer.

"Mi intención es que los alumnos se puedan examinar a sí mismos y ver cómo salen (...) les dejaría hacerlo una y otra vez hasta que tengan 100 para que entiendan..." (Michal); "Nuestro aprendizaje se basa en la comunicación entre nosotros y en generar confianza en nosotros [los maestros]” (Rachel).

\footnotetext{
${ }^{11}$ Todos los nombres son ficticios a los efectos de mantener la confidencialidad de la información.
}

Dorfsman, M. \& Horenczyk, G. El cambio pedagógico en la docencia universitaria en los tiempos de Covid-19 
En síntesis, en esta primera categoría se pueden identificar básicamente tres enfoques; el de los docentes enfocados en su propia enseñanza (TCA), aquellos enfocados en el aprendizaje de los estudiantes (SCA) y los centrados en la estructura epistemológica del contenido a enseñar (CCA).

En la literatura científica se encuentran indicios acerca de la relación entre los docentes que responden a la SCA y la disposición a utilizar tecnologías (Brown, 2003; Emaliana, 2017; Kálmán et al., 2020). En el próximo tópico analizaremos modelos de respuesta de docentes acerca de la inclusión de TIC en situación de crisis.

\section{La inclusión de las TIC en la enseñanza}

Los autores se refieren a las TIC en un sentido amplio, como herramientas y entornos digitales, creados para la enseñanza, o adaptados y-o recuperados para ella.

Esta categoría incluye respuestas que dieron los docentes a la pregunta sobre el uso de tecnologías. En sus respuestas, los docentes se refirieron tanto al período lectivo bajo CRTS, como al anterior y posterior. En general, la mayoría de los docentes coincidieron en un uso de herramientas tecnológicas de baja intensidad previo al CRTS: coincidiendo en el uso de Power-Point o en un uso mínimo de la plataforma Moodle.

Del análisis cuantitativo se verifica que, en una primera reacción a la situación de enseñar en línea, sólo un $9 \%$ de los docentes informaron que poseían ya experiencia previa en enseñanza en línea y que, por lo tanto, podían seguir haciéndolo; un 3\% indicó que no podía o no estaba dispuesto a hacerlo de ninguna manera; un $29 \%$ declaró que podía enseñar en línea pero que no le sería satisfactorio; y un $40 \%$ declaró estar "abiertos a la oportunidad" de aprender nuevas herramientas o de innovar pedagógicamente.

Cuando los docentes fueron consultados sobre su sensación de éxito o no en sus primeras clases en línea, el $85 \%$ las consideraron desde razonables hasta muy exitosas. Cuando fueron consultados acerca de las razones de este "éxito", $43 \%$ de los docentes atribuyeron su éxito al tiempo de preparación invertido; $31 \%$ al soporte tecno-pedagógico recibido; $31 \%$ a una sensación de urgencia ("no hay otra alternativa"), y el 39\% a sus conocimientos y destrezas tecnológicas.

A partir del análisis de los datos se identificó que, por un lado, un porcentaje significativo de docentes dijo tener conocimientos y destrezas tecnológicas $(33 \%)^{12}$, pero que no las utilizaba en sus prácticas; por el otro, un porcentaje similar de docentes dijo estar abierto a nuevos aprendizajes e innovaciones $(34 \%)^{13}$. Estos primeros datos subrayaron la necesidad de profundizar, en las entrevistas cualitativas, si existía alguna relación entre el uso previo de tecnologías en la enseñanza y la posibilidad de adaptarse a los cambios impuestos por la emergencia. Este interrogante es el que guió el posterior análisis.

En el análisis cualitativo, se identificaron tres subgrupos de profesores:

Un primer grupo es el de los profesores que utilizaron previamente tecnologías, y durante el CRTS lo intensificaron. Estos docentes en general, han adoptado una posición favorable para mantener lo aprendido y extenderlo en sus prácticas futuras:

${ }^{12}$ El 39\% entre el $85 \%$ del total de los docentes que indicaron que su enseñanza fue exitosa.

${ }^{13}$ El $40 \%$ entre el $85 \%$ del total de los docentes que indicaron que su enseñanza fue exitosa.

Dorfsman, M. \& Horenczyk, G. El cambio pedagógico en la docencia universitaria en los tiempos de Covid-19

Page 11 of 27 
"Ahora con la Corona usamos muchos elementos de Moodle, fue muy bueno ya que esto también nos obligó a estudiar mucho sobre Moodle, cosas que armamos, todo tipo de cuestionarios que hicimos, viendo que todos los alumnos están con nosotros y nos siguen" (Elisheva); "Es importante aclarar que ya usamos estas herramientas antes de la Corona en el curso (...), y luego también intentamos usar herramientas que también sean “agradables", y lo hicimos" (Rachel).

A este grupo se lo ha denominado "experimentados". Se trata de docentes que no necesariamente manejan todas las herramientas digitales; de hecho, la mayoría de ellos tuvo que aprender las nuevas herramientas propuestas por la universidad - Zoom y Panopto $^{14}$ - pero se desprende de sus historias que pudieron aprenderlas fácilmente ya que estaban alfabetizados digitalmente (Eshet-Alkalai, 2004). En este primer grupo, y según el modelo TAM (Venkatesh \& Bala, 2008; Venkatesh \& Davis, 2000), se identificaron docentes que percibieron la utilidad del uso de las tecnologías, y también su facilidad de uso.

Un segundo grupo está formado por docentes que, antes de CRTS, hacían un uso mínimo de herramientas digitales. Todos ellos habían hecho un uso muy limitado de Moodle, y en general coincidieron en el uso de Power-Point como ayuda didáctica y herramienta visual. Los integrantes de este grupo, como Meital y Moshe, expresaron haber "descubierto" de alguna manera, el mundo de las herramientas digitales, y estarían dispuestos a utilizarlo:

"... Entonces, por un lado, estaba muy satisfecha, yo estaba ... como un niño con un juguete nuevo, iwaw!" (Meital). "Descubrí que hay realmente un mundo rico y lleno de cosas, y comencé a estudiar qué es Panopto, cómo se usa y zoom antes de esto. .. todos dicen zoom, zoom, zoom y antes de eso nunca supe que existía algo, entonces sí ... (Moshe).

El esfuerzo básico de estos docentes fue dominar las tecnologías para poder llevar sus prácticas conocidas a nuevos entornos o poder implementar nuevas prácticas.

En este grupo, el impacto de enseñar con Zoom y a distancia los puso en una situación casi de "primera vez" para el uso de estas nuevas tecnologías:

“...pero... yo estaba ... no puedo decirles que no me sentí presionada. Nunca usé el zoom, no sabía cómo iba a lucir y al mismo tiempo estaba en casa con mis hijos pequeños (...) luego usando y aprendiendo a través de "prueba y error" y trabajando con mis colegas... "(Revital) [antes de este período] .... tenía una especie de barrera, no, no haces esto, tienen que venir a mi oficina o no sé entonces creo que va a haber algún cambio $^{15 "}$ (Moshe).

Algunos profesores afirmaron que la experiencia posibilitó la caída de una barrera psicológica:

“... voy a tener menos de esta barrera y es muy significativo, porque sabes, a veces también hay [años] sabáticos o de repente hay que enseñar... es decir, es una barrera

\footnotetext{
${ }^{14}$ Panopto es una plataforma para la producción, diseño y almacenamiento de videos digitales. Para más información ver https://www.panopto.com/

${ }^{15} \mathrm{El}$ entrevistado asume que se puede recibir a los alumnos vía zoom y no sólo en la oficina.
}

Dorfsman, M. \& Horenczyk, G. El cambio pedagógico en la docencia universitaria en los tiempos de Covid-19

Page 12 of 27 
psicológica que de repente me di cuenta que tenía y que creo que ya no la tendré, y que cuando necesite enseñar [vía zoom], podré hacerlo" (Revital).

En este segundo grupo, y según el modelo TAM3, se han identificado profesores que han "descubierto" la utilidad del uso de las tecnologías y sienten placer por haberlo hecho, aunque todavía no las consideran fáciles de usar. A este grupo se lo ha denominado "entusiastas".

Un tercer grupo es el de los docentes que han debido incorporar herramientas tecnológicas a raíz del CRTS, pero mostraron menos entusiasmo por ellas y por su eventual uso posterior.

En este grupo, se encuentran, por ejemplo, Yael y Lior. Lior mencionaba el PPT y eventualmente algún uso del formulario de Google, más allá de un uso menor y necesario de moodle y zoom. Al consultarle sobre la vuelta a la práctica, destacaba específicamente la flexibilidad y comodidad de la herramienta, pero no algún tipo de ventaja pedagógica: "Puedo ser flexible pero, por otro lado, prefiero la enseñanza frontal, pero también veo ventajas, las ventajas de enseñar con zoom son básicamente el tiempo porque me lleva al menos una hora llegar a la universidad cada vez..." (Lior). Yael se vio muy afectada por su experiencia negativa con zoom, particularmente por el problema de las "cámaras cerradas”. Ella informaba, sobre el eventual regreso a clases: “... a los estudiantes les gusta mucho (zoom). Desde mi punto de vista es malo, porque soy menos libre y auténtica, yo... no hay nada que hacer, tú lo viste, soy menos libre, no sé... es... soy menos libre... "(Yael)

A este grupo se lo ha denominado "cautelosos" y, según el modelo TAM3, la utilidad o facilidad de uso de las tecnologías no estaría clara para este tipo de docentes.

En resumen, los autores han identificado bajo este segundo tema tres subgrupos de profesores: los experimentados, los entusiastas y los cautelosos. Sin duda, la experiencia forzada de la enseñanza en línea ha generado en cada uno de estos grupos una percepción particular sobre el uso de las tecnologías y con ello, un impacto diferente en cuanto a la posibilidad de continuar con su uso luego de la CRTS.

\section{La confrontación con la CRTS}

En este segundo tema, los investigadores indagaron acerca de la reacción de los docentes a la necesidad de comenzar a enseñar en línea.

En la fase cuantitativa, en la segunda sección del cuestionario, los docentes debían completar la siguiente frase: "Cuando escuché acerca de la transición a la enseñanza en línea, yo pensé..."

La Tabla 1 muestra el porcentaje de respuestas obtenido a cada opción presentada.

Dorfsman, M. \& Horenczyk, G. El cambio pedagógico en la docencia universitaria en los tiempos de Covid-19 
Tabla 1: Primeras reacciones al cambio en la enseñanza

\begin{tabular}{|c|c|c|}
\hline & Absolutamente, no puedo enseñar en línea & $1.35 \%$ \\
\hline 2. & Soy capaz de enseñar en línea pero no tengo la intención de hacerlo. & $1.81 \%$ \\
\hline 3. & Puedo enseñar en línea, pero será menos satisfactorio para mí. & $28.67 \%$ \\
\hline 4. & $\begin{array}{l}\text { No puedo enseñar en línea de inmediato, pero tendré éxito si recibo } \\
\text { el apoyo adecuado. }\end{array}$ & $14.00 \%$ \\
\hline 5. & Ya tengo experiencia en la enseñanza online, me conviene. & $4.06 \%$ \\
\hline & $\begin{array}{l}\text { Ya tengo experiencia en la enseñanza en línea, pero me temo que los } \\
\text { estudiantes no están preparados para este tipo de desafío. }\end{array}$ & $4.74 \%$ \\
\hline 7. & $\begin{array}{l}\text { No tengo experiencia en la enseñanza en línea, pero esta es una } \\
\text { oportunidad para aprender a usar nuevas herramientas. }\end{array}$ & $24.15 \%$ \\
\hline & $\begin{array}{l}\text { No tengo experiencia en la enseñanza en línea, pero esta es una } \\
\text { oportunidad para la innovación pedagógica. }\end{array}$ & $16.03 \%$ \\
\hline & Otras & $5.19 \%$ \\
\hline
\end{tabular}

Fuente: datos obtenidos en el cuestionario suministrado a los docentes universitarios en la fase cuantitativa de esta investigación.

El 32\% informó sobre su incomodidad para enseñar en línea (opciones 1,2,3), un 14\% indicó que podría hacerlo si recibiese un adecuado soporte (opción 4), y un 49\% mostró una buena predisposición para hacerlo (opciones 5,6,7,8).

Obtenidos estos primeros datos, en la fase cualitativa los investigadores trataron de comprender el significado de cada una de estas reacciones a fin de constatar la existencia de posibles relaciones entre la percepción de la emergencia y la disposición al cambio frente a ella.

En la entrevista, los docentes informaron sobre reacciones afectivas de diversa índole: sorpresa, miedo, angustia, preocupación, entusiasmo. Finalizado el primer impacto, se los inquirió acerca de cómo seguir adelante.

Una parte de ellos (principalmente, los "experimentados") comprendieron la profundidad del cambio, e informaron sobre la decisión de cambiar el enfoque pedagógico del curso de manera significativa, a partir de una "decisión estratégica";

... "Tomé una decisión estratégica, que es grabar las clases de antemano” (Michal).

Esta profesora explicaba que produjo videos de hasta 15 ', según los "fundamentos de la educación a distancia" y un método similar al del "aula invertida" sumando las horas de recepción de alumnos (vía zoom). Otros profesores probaron prácticas similares, o prácticas intensivas en Moodle y trabajo en grupo cuya presentación llegó "con la cámara abierta". Rachel informaba que cambió su práctica de manera significativa:

“... chat, Moodle, foro en Moodle, abrimos un foro adicional para actividad adicional que finalmente les dio (a los estudiantes) la posibilidad de ver series israelies y reaccionar ante ellas, y les dieron puntos extra" (Rachel).

Los profesores "experimentados", a diferencia de los "entusiastas" y "cautelosos", estaban más familiarizados con el lenguaje digital. Estos docentes entendieron que, para seguir adelante, debían modificar su práctica y, de hecho, muchos de ellos lo hicieron. Estos docentes entendieron que es necesario reajustar sus prácticas a los nuevos entornos

Dorfsman, M. \& Horenczyk, G. El cambio pedagógico en la docencia universitaria en los tiempos de Covid-19 
tecnológicos. Por ejemplo, Shlomo adoptó el modelo de aula invertida, después de varios intentos en los que priorizó la "prueba y error":

"Dije que sería mucho más controlado hacer una lección invertida dedicada al material y luego hacer una sesión de preguntas y respuestas con los estudiantes, y ese era el formato..." (Shlomo).

Se puede considerar que estos profesores experimentados fueron, además, "estratégicos".

El grupo de profesores "entusiastas", y en menor medida los "cautelosos", debían dedicar la mayor parte de su tiempo a "adquirir" un lenguaje nuevo e inexplorado para ellos, el "lenguaje digital" (Eshet-Alkalai, 2004). En algunos casos, expresaron interés y entusiasmo; en otros, lo hicieron por falta de otra alternativa. Estos docentes expresaron preocupación por el "tiempo excesivo" que se requería para preparar las clases, y la sensación de "enseñar menos contenido" en las mismas, en comparación con la educación convencional (Le Fevre, 2014).

Esta afirmación recibió también respaldo en los datos del análisis cuantitativo, en el cual se vio que $43 \%$ de los docentes consultados señalaba "la excesiva cantidad de tiempo invertido en la preparación de las clases" como factor de éxito. Los investigadores estimaron que el tiempo puede ser considerado más un obstáculo que un favorecedor de inclusión de tecnologías a futuro.

Una vez que los docentes percibían que podían "dominar" el entorno tecnológico, el esfuerzo principal estaba orientado a cómo podrían enseñar en este nuevo entorno tal como lo hacían anteriormente.

Un ejemplo de esto lo muestra Michael:

"Traté de hacer cambios significativos en el curso, y los hice, a lo largo de tres o cuatro clases sobre las que, luego, recibí una crítica muy positiva de los estudiantes sobre esa parte del curso - pero, nuevamente, después de la cuarta clase me di cuenta de que llegué a ... no sé ... 5\% del material que me había propuesto para el semestre; y luego fui a clases totalmente comunes con algunas adaptaciones "tácticas" (Michael).

Estos docentes entendieron que era necesario utilizar los nuevos entornos tecnológicos, pero lo hicieron mayormente con sus prácticas convencionales anteriores. A estos docentes, que pueden ser o bien entusiastas o cautelosos, se los puede caracterizar también como "tácticos".

\section{El impacto del CRTS en las prácticas pedagógicas de los docentes}

En la tercera parte del cuestionario, se preguntó a los docentes acerca del "día después".

Ante la pregunta: ¿"Piensa que esta experiencia va a afectar su práctica pedagógica?, un $44 \%$ respondió que no, o en muy poca medida; mientras que el 56\% respondió que sí, en moderada o en gran medida.

Al ser consultados sobre su auto-percepción como docentes, el $47 \%$ reportó que está en condiciones de responder a las expectativas de los estudiantes; el $42 \%$ dijo estar en condiciones de utilizar medios digitales a fin de sostener la calidad de la enseñanza; y el $46 \%$ indicó que es flexible para readecuar sus programas en caso de necesidad.

Dorfsman, M. \& Horenczyk, G. El cambio pedagógico en la docencia universitaria en los tiempos de Covid-19

Page 15 of 27 
Adicionalmente, el $68 \%$ informó que no estaba interesado o en muy pequeña medida, en incorporar la modalidad de clases vía zoom a sus cursos; y sólo un $19 \%$ se mostró interesado en convertir sus cursos presenciales en cursos en línea.

Finalmente, al ser consultados sobre sus necesidades en una posible crisis futura, el $38 \%$ de los docentes señaló la necesidad de contar con más tiempo para prepararse, mientras que el $37 \%$ mencionó la necesidad de contar con soporte tecno-pedagógico para preparar sus clases.

De estas primeras respuestas se desprende que la mayoría de los docentes universitarios considera que su práctica se ha visto impactada (56\%), siendo que buena parte de ellos se siente en condiciones de utilizar medios digitales, responder a las necesidades de sus estudiantes y readecuar sus programas. Esto, sin necesidad de convertir a sus cursos en cursos en línea mediante el uso de sistemas de videoconferencia.

En la fase cualitativa, los investigadores profundizaron en estos aspectos, en particular en la posibilidad y naturaleza del cambio en las prácticas pedagógicas. Ahora bien, cuando se indagó a los docentes acerca de esta temática, sus respuestas reflejaron un cambio en la forma de pensar las prácticas en el futuro, sin que quede claro si se podría instrumentar. Entonces, si bien no fue posible comprobar si habría un cambio efectivo en las prácticas en las aulas, sí fue posible discernir si los docentes pensaban en ellas de forma diferente a lo que las pensaban antes del CRTS, con lo cual estaríamos hablando de un cambio en la concepción pedagógica más que de un cambio en las prácticas en el aula a futuro.

Los docentes informaron sobre dos tipos de cambio: por un lado lo que parecería ser un cambio formal en la enseñanza; por el otro un cambio genuino en la enseñanza y en su enfoque (Fullan, 2002; Le Fevre, 2014; Tarling \& Ng'ambi, 2016).

Ejemplo del primero es el caso de Revital: “

...estoy bastante segura de que como existe esta herramienta [Panopto], lo más probable es que no requiera asistencia [a clases], y voy a permitir que los alumnos vean las clases grabadas en el sitio de Moodle..., en la medida que prefieran y no puedan acceder a él, y desde mi punto de vista esto me quita mucha presión por el hecho de controlar la asistencia... Y voilá, esto es para mí [lo que puede cambiar] (Revital].

Ejemplo del segundo es el caso de Elisheva:

“... creo que esto va a cambiar totalmente nuestra forma de enseñar porque realmente todo este tiempo "dimos la papilla masticada "a los alumnos; Y este año no lo hicimos así y se enfrentaron (solos) con el material, y aunque hubo algunas quejas, se ve que están muy satisfechos y tienen altas notas y trabajan a muy buen nivel y creo que, en definitiva, creo que más importante para mí es que conozcan el contenido y lo estudien de verdad que en darles lo que quieren en bandeja de plata, asi me parece" (Elisheva).

Se identificaron entonces dos grandes grupos de docentes: aquellos que pretendían producir un cambio formal; y quienes proponían cambios profundos en sus prácticas docentes.

Un tercer grupo, que se suma a los anteriores, es el de los que no han sido impactados y no pretenden incluir cambios en sus prácticas: Yael, por ejemplo, diferencia claramente entre enseñar en tiempos de crisis y enseñar en tiempos normales:

Dorfsman, M. \& Horenczyk, G. El cambio pedagógico en la docencia universitaria en los tiempos de Covid-19

Page 16 of 27 
“... durante la crisis es lo mejor que nos pudo pasar porque nos salvó. En la vida normal, no lo veo..." "(Yael);

Marc, con el mismo espíritu, dice:

"Creo que aprendí a aprender todo tipo de herramientas en línea por la fuerza, pero las clases las daré con gusto de forma frontal..." (Marc); y Lior: “... puedo ser flexible, pero por otro lado prefiero enseñar frontalmente..." (Lior).

En síntesis, es dable identificar tres grupos de profesores: El primero de ellos es el de los "profesores experimentados", que están dispuestos a realizar cambios claros y significativos en sus prácticas. En general, se trata de profesores que se centran básicamente en sus alumnos, que ya utilizaban tecnologías antes del CRTS, y entienden que sus prácticas deben adaptarse a los entornos tecnológicos. Estos profesores intentan realizar cambios significativos, que van desde la forma de organizar las clases hasta la forma de concebir cómo aprenden los estudiantes en esas clases; Parecen ser profesores que han cambiado su enfoque, de uno centrado en el profesor a uno más centrado en el alumno (Brown, 2003; Kember \& Kwan, 2000; Tarling \& Ng'ambi, 2016). Estos "profesores experimentados" serían aquellos que, según nuestro análisis cuantitativo, ya tienen conocimientos previos de tecnologías digitales y ya han adoptado el lenguaje digital ${ }^{16}$.

El segundo grupo es el de los "profesores entusiastas". Se trata de profesores interesados en algún tipo de cambio. Son los docentes que, si bien no tenían mucha experiencia previa en el uso de las tecnologías, con la crisis descubrieron un nuevo mundo y estarían interesados en aprovecharlo. Estos profesores mantuvieron, en general, sus prácticas pedagógicas previas en los nuevos entornos, y los cambios en los que habrían pensado eran cambios formales; o cambios aun indefinidos. En general, se trata de profesores centrados en los contenidos o en sí mismos, que sin embargo han descubierto las posibilidades que les brinda el mundo de las tecnologías (Admiraal et al., 2017; Alenezi, 2017; Beetham \& Sharpe, 2007; Blau \& Peled, 2012). Estos docentes, según el análisis cuantitativo, se encuentran entre los que están "abiertos a la oportunidad ${ }^{17}$ "; no es seguro que tengan conocimientos previos de herramientas digitales o, bien, si conocían, no estaba claro que estuviesen dispuestos a utilizarlas en la enseñanza.

Finalmente, se ha identificado un tercer grupo de docentes, el de los "profesores cautelosos", aquellos que valoran el uso de la tecnología en tiempos de emergencia, pero no la consideran adecuada para tiempos "normales" y no están evaluando cambios en sus prácticas. En el análisis cuantitativo, son los que no mostraron voluntad de cambiar sus prácticas pedagógicas ${ }^{18}$.

Estos perfiles docentes responden a tres ejes interrelacionados: 1. La concepción pedagógica; 2. el nivel de alfabetización digital y 3. la calidad del cambio pedagógico. Los investigadores han denominado a esta tipología; "Tipología de Aceptación del

\footnotetext{
${ }^{16}$ Alrededor de un $33 \%$ que informaron sobre su conocimiento y destrezas tecnológicas.

${ }^{17}$ Pueden identificarse como el $40 \%$ de los docentes, que informaron estar abiertos a nuevos aprendizajes e innovaciones, de acuerdo a lo obtenido en Tabla 1.

18 Pueden identificarse como el $32 \%$ de los consultados que informaron su incomodidad para enseñar en línea, de acuerdo a lo obtenido en la Tabla 1.
}

Dorfsman, M. \& Horenczyk, G. El cambio pedagógico en la docencia universitaria en los tiempos de Covid-19

Page 17 of 27 
Cambio Pedagógico" (TACP), que queda reflejada en la tabla 2. En esta tabla se refleja el análisis cualitativo realizado de acuerdo con cada una de las subcategorías obtenidas.

Tabla 2: Tipología de Aceptación del Cambio Pedagógico (TACP)

\begin{tabular}{|c|c|c|c|c|}
\hline Perfiles & & $\begin{array}{l}\text { Concepción } \\
\text { pedagógica }\end{array}$ & $\begin{array}{l}\text { Alfabetización } \\
\text { digital }\end{array}$ & $\begin{array}{l}\text { Cambio } \\
\text { pedagógico }\end{array}$ \\
\hline \multirow[t]{5}{*}{ Experimentados } & Michal & 3 & 3 & 3 \\
\hline & Rachel & 3 & 3 & 3 \\
\hline & Elisheva & 3 & 3 & 3 \\
\hline & Shlomo & 3 & 2 & 3 \\
\hline & Esther & 2 & 3 & 3 \\
\hline \multirow[t]{7}{*}{ Entusiastas } & Revital & 3 & 3 & 2 \\
\hline & Noa & 1 & 3 & 2 \\
\hline & Michael & 2 & 2 & 2 \\
\hline & Meital & 2 & 2 & 2 \\
\hline & Moshe & 2 & 2 & 2 \\
\hline & Shulamit & 1 & 2 & 2 \\
\hline & Avigdor & 1 & 2 & 2 \\
\hline \multirow[t]{3}{*}{ Cautelosos } & Yael & 2 & 1 & 1 \\
\hline & Marc & 1 & 2 & 1 \\
\hline & Lior & 1 & 1 & 1 \\
\hline \multirow{3}{*}{\multicolumn{2}{|c|}{ Referencias }} & $\begin{array}{l}\text { 1. Centrado } \\
\text { en el } \\
\text { docente }\end{array}$ & $\begin{array}{l}\text { 1.Usa } \\
\text { herramientas } \\
\text { digitales, no se } \\
\text { entusiasma en } \\
\text { seguir usando }\end{array}$ & $\begin{array}{l}\text { 1. Resiste el } \\
\text { cambio / } \\
\text { cambio táctico }\end{array}$ \\
\hline & & $\begin{array}{l}\text { 2. Centrado } \\
\text { en el } \\
\text { docente } \rightarrow \\
\text { Centrado en } \\
\text { el alumno } \\
\end{array}$ & $\begin{array}{l}\text { 2. Descubrió las } \\
\text { herramientas, se } \\
\text { interesa en } \\
\text { seguir usando. }\end{array}$ & $\begin{array}{l}\text { 2. Acepta } \\
\text { cambiar, } \\
\text { cambio formal }\end{array}$ \\
\hline & & $\begin{array}{l}\text { 3. Centrado } \\
\text { en el } \\
\text { alumno }\end{array}$ & $\begin{array}{l}\text { 3. ya usaba } \\
\text { herramientas } \\
\text { digitales, } \\
\text { continua. }\end{array}$ & $\begin{array}{l}\text { 3. Cambio } \\
\text { genuino }\end{array}$ \\
\hline
\end{tabular}

Fuente: basado en los resultados del análisis cualitativo de esta investigación

\section{La perspectiva institucional}

En esta categoría, el análisis fue inverso: habiendo identificado los tres perfiles pedagógicos, fue posible examinar cómo los docentes en cada uno de estos tres grupos percibieron el apoyo institucional durante el CRTS.

Los docentes experimentados valoraron el apoyo institucional pero no lo consideraron imprescindible:

Dorfsman, M. \& Horenczyk, G. El cambio pedagógico en la docencia universitaria en los tiempos de Covid-19

Page 18 of 27 
"Desde mi perspectiva, estoy muy satisfecho, no necesito nada más en este momento" (Michal, experimentado).

Los cautelosos, por su parte, intentaron sobreponerse sin la ayuda, aunque también valoraron el hecho de que disponían de apoyo:

"Nos las arreglamos solos la mayor parte del tiempo, pero cuando [necesitábamos] [ayuda], creo que había algo. ... Había apoyo y estábamos todos en el mismo barco" (Marc, cauteloso).

Los entusiastas finalmente consideraron que el apoyo institucional era crucial para intentar cualquier cambio profundo:

"Pero realmente un acercamiento de la institución (...), digamos, ¿vale? Y no es solo una cosa, sino que debería haber una atmósfera que realmente estamos cambiando aqui" (Meital, entusiasta).

En resumen, al analizar las primeras cuatro categorías temáticas definidas, fue posible identificar tres perfiles pedagógicos. La quinta categoría temática ha hecho posible enriquecer estos perfiles, analizando la percepción de cada grupo sobre el apoyo institucional.

En la próxima sección se confrontarán estos datos con el modelo UTAUT, de aceptación de tecnologías por parte de los docentes.

\section{Cambio pedagógico y aceptación de uso de las tecnologías}

A fin de profundizar en la comprensión acerca de la naturaleza del cambio pedagógico en los docentes, es posible aprender del modelo de Venkatesh (2003) acerca de la aceptación de tecnologías por parte de docentes. Se considerarán las cuatro categorías centrales en dicho modelo, ya mencionadas anteriormente: La "expectativa de desempeño", la "expectativa de esfuerzo", la "influencia social" y las "condiciones facilitadoras" (Venkatesh et al., 2003).

Al combinar las cuatro categorías con la TACP, es dable determinar en qué medida cada categoría es relevante para cada uno de los perfiles identificados. La tabla 3 muestra los resultados obtenidos:

Dorfsman, M. \& Horenczyk, G. El cambio pedagógico en la docencia universitaria en los tiempos de Covid-19 
Tabla 3: Modelo de Aceptación de Tecnologías Actualizado

\begin{tabular}{|c|c|c|c|}
\hline $\begin{array}{l}\text { Categorías de } \\
\text { aceptación de } \\
\text { tecnologías / TACP }\end{array}$ & Experimentados & Entusiastas & Cautelosos \\
\hline $\begin{array}{c}\text { Expectativas de } \\
\text { rendimiento }\end{array}$ & Alta relevancia & Alta relevancia & No relevante \\
\hline $\begin{array}{c}\text { Expectativas de } \\
\text { esfuerzo }\end{array}$ & Baja relevancia & Alta relevancia & Alta relevancia \\
\hline $\begin{array}{c}\text { Influencia Social } \\
\text { pedagógicas }\end{array}$ & $\begin{array}{c}\text { No relevante } \\
\text { "enfoque centrado en el } \\
\text { estudiante" }\end{array}$ & $\begin{array}{c}\text { Alta relevancia } \\
\text { alta: De un "enfoque } \\
\text { centrado en el } \\
\text { docente" a un } \\
\text { el estudiante " }\end{array}$ & $\begin{array}{c}\text { No relevante: } \\
\text { enfoque centrado en el } \\
\text { docente" }\end{array}$ \\
\hline $\begin{array}{c}\text { Condiciones } \\
\text { facilitadoras }\end{array}$ & Baja relevancia & Alta relevancia & No relevante \\
\hline & & No relevante \\
\hline
\end{tabular}

Fuente: basado en los resultados del análisis cualitativo de esta investigación.

Los profesores experimentados son conscientes del rendimiento que les van a proporcionar las tecnologías; conocen la utilidad y no dudan de los resultados a obtener:

"Ahora con la corona usamos muchas características del modelo, fue realmente bueno porque también nos obligó a aprender muchas cosas en el modelo, cosas que hicimos por ellos, todo tipo de cuestionarios que completamos mientras veíamos eso todos los estudiantes están con nosotros y nos siguen_" (Elisheva).

A esto se suma la voluntad de trabajar en modo de "prueba y error", que se vuelve típico de este grupo:

“... y también al grabar, y con la pizarra y cosas por el estilo así que me tomó un tiempo hasta que intenté colocar la computadora en todo tipo de lugares en esta sala y ver a qué

Dorfsman, M. \& Horenczyk, G. El cambio pedagógico en la docencia universitaria en los tiempos de Covid-19 
horas el sol no debería reflejarse y ... fue realmente un ensayo y mucho error... "(Shlomo).

Asimismo, si bien la expectativa de desempeño fue alta, la expectativa de esfuerzo mostró poca relevancia porque no es una variable problemática, ya que la alfabetización digital que han adquirido les da una sensación de seguridad y tranquilidad;

“... En resumen, ya habíamos estado usando herramientas digitales relativamente extensas incluso antes de Corona. Como si todos lo supiéramos y también pensáramos que es importante y bueno usarlo" (Rachel).

Estos profesores no se preocupan por los factores sociales que se incluyen en la categoría de influencia social, y consideran que el apoyo que reciben de la institución es relevante pero no determinante.

"Estoy muy satisfecha, no necesito nada más allá de esta etapa ..." (Michal). "En general sentí que la institución quería dar más de lo que yo quería recibir, lo que significa que realmente había mucho contenido" (Esther).

Los profesores entusiastas, a diferencia de los anteriores, no conocían específicamente el posible desempeño de las tecnologías, pero una vez que las descubrieron, les generó expectativas de uso; por lo que este factor también es muy relevante para ellos.

"No le tengo miedo a estas cosas, no soy nada tecnofóbica. De hecho pienso ... y de hecho, dije 'wow que divertido, finalmente aprendiendo cosas que son bonitas y nunca tuve tiempo ..." (Meital);

"luego intentaba a través de ensayo y error con compañeros" (Revital).

A diferencia de los anteriores, la facilidad de uso aún no es obvia, pero es una barrera de cambio. Por lo tanto, la expectativa de esfuerzo es un factor de alta relevancia para ellos. En cuanto a los factores sociales, son conscientes de ellos ya que les brindarán un sentido de apoyo colectivo para el cambio; de la misma manera con el apoyo institucional que es parte de la viabilidad misma del cambio;

“... necesitamos cambiar todo aquí, así que incluso si quiero hacerlo, no quiero que sea solo con mis estudiantes, no quiero ser el profesor "odiado" que da muchas tareas, ¿de acuerdo? (Meital).

Por último, facilitarles las condiciones también es muy relevante para ellos, ya que en esto se basa el posible cambio:

"Entonces respecto de la cuestión técnica, creo que la universidad hizo un movimiento muy acertado, y creo que realmente hizo todo lo posible para reducir el daño o minimizar el daño ..." (Noa).

Por último, los "profesores cautelosos", no parecen responder a ninguna de las cuatro categorías del modelo de aceptación de tecnologías mencionadas anteriormente: no consideran la utilización de las tecnologías como una posibilidad cierta dado que su implementación es muy complicada para ellos;

"No soy muy tecnológica, y me estresó ... sé qué, abrir aquí, abrir allá, pero bueno, pasó ... (Yael) ...".

Dorfsman, M. \& Horenczyk, G. El cambio pedagógico en la docencia universitaria en los tiempos de Covid-19 
No perciben la existencia de un consenso social para el cambio o no lo ven relevante para ellos, y no esperan apoyo institucional al respecto, aunque saben que lo tendrán en caso de necesidad;

"Creo que en ese sentido no ... manejamos la mayor parte del tiempo solos, pero cuando fue necesario creo que hubo algo ... hubo apoyo y todos estaban en el mismo barco ..." (Marc).

Los autores proponen agregar una quinta categoría al modelo aplicado específicamente a docentes, la "expectativa pedagógica". Esta categoría expresa la medida en que los docentes consideran que la implementación de un cambio basado en las TIC puede ser favorable o no para los estudiantes. La misma está claramente relacionada con el enfoque de cada grupo, desde el enfoque centrado en el profesor - que caracteriza al grupo de los profesores cautelosos, hasta el enfoque centrado en el estudiante, que caracteriza a los experimentados.

De este modo, a diferencia de la visión habitual sobre la introducción de tecnologías en las aulas (ver por ejemplo:(Bebell \& Kay, 2010; Blau \& Peled, 2012; Graves \& Bowers, 2018; McLoughlin \& Lee, 2008; Terhart, 2013) según la cual el uso de computadoras contribuyó a que la mayoría de los docentes reportaran cambios en la enseñanza gracias a un proceso planificado de trabajo continuo de apoyo y formación, en estos casos el cambio se dio en situaciones de presión, no planificadas y con formación docente limitada.

\section{Discusión}

El 10.3.2020, las autoridades de la Universidad Hebrea anunciaban que la apertura del semestre, prevista para el 15.3.2020, se pospondría una semana y que la enseñanza durante ese semestre se realizaría a través de la enseñanza en línea, tanto para profesores como para estudiantes. De este modo, posiblemente sin saberlo, se producía uno de los cambios más significativos en la enseñanza universitaria de los últimos años.

$\mathrm{Al}$ iniciar este trabajo los investigadores se plantearon las siguientes preguntas: ¿En qué medida una situación de crisis puede impactar en la disposición del docente universitario a efectuar cambios pedagógicos en su práctica?

Ante la situación de crisis, y habiendo examinado lo sucedido en la primera etapa del COVID-19, que es lo que se considera la emergencia misma, los autores se preguntan por el impacto de esta situación en las prácticas y concepciones pedagógicas de los docentes universitarios.

La literatura de investigación refiere ampliamente al concepto de cambio en la enseñanza: cambio profundo y cambio formal, cambio estructural y cambio cultural, cambio genuino y cambio forzado (Admiraal et al., 2017; Brown, 2003; Fullan, 2002; Schreurs \& Dumbraveanu, 2014).

Como se afirmaba previamente, se han identificado tres tipos de actitudes de los docentes hacia el cambio: En primer lugar, los que han cambiado su práctica durante el CRTS y tienen la intención de continuarla, es la actitud de los "profesores experimentados". En segundo lugar, los que han cambiado formalmente su práctica y están interesados en algún tipo de cambio en el futuro, es la actitud de los "profesores entusiastas". Por último,

Dorfsman, M. \& Horenczyk, G. El cambio pedagógico en la docencia universitaria en los tiempos de Covid-19

Page 22 of 27 
aquellos que han cambiado formalmente sus prácticas durante el CRTS, pero no están dispuestos a continuarlo, que parece ser la actitud de los "profesores cautelosos".

Cada uno de estos tres grupos concebían el cambio de manera diferente. Los "profesores experimentados" lo percibían como parte de una decisión estratégica, lo que da cuenta de un cambio en su concepción de cómo aprenden los estudiantes. Ellos ya han pasado por un proceso de alfabetización digital que les permitió acceder a entornos digitales conocidos o nuevos en mejores condiciones (Eshet-Alkalai, 2004; Wood, Mueller, Willoughby, Specht, \& Deyoung, 2005), y se han sentido más preparados para afrontar positivamente la situación de emergencia. Estos docentes conciben esta situación como una oportunidad luego de la cual podrían realizar cambios en sus prácticas, a partir de los cambios experimentados en su concepción de la enseñanza.

Los "profesores entusiastas" estaban interesados en el cambio, pero, o no sabían exactamente cómo realizarlo o no distinguían entre el cambio formal y el cambio genuino, y sólo estarían preparados para un cambio formal. Por ahora son "tácticos". y "se han unido" al mundo digital en el marco de esta crisis. Por último, los "profesores cautelosos" - también "tácticos " - experimentaron la CRTS como una situación de emergencia (Hodges et al., 2020; Murphy, 2020) que debía ser superada, lo que implicaba un cambio temporario en sus prácticas y un volver a la "normalidad", después de la misma.

Claramente, la caracterización de los entrevistados en cada una de las categorías no implica un enfoque determinista o dicotómico. El propósito de la tipología es comprender conceptualmente los rasgos predominantes de los diferentes perfiles, con el fin de identificar aspectos destacados en cada uno que nos permitan establecer líneas de acción.

En la segunda parte del análisis, se utilizó la tipología propuesta, TACP, para analizar el modelo UTAUT a fin de profundizarlo y ampliarlo (Admiraal et al., 2017; Venkatesh \& Bala, 2008; Venkatesh \& Davis, 2000; Venkatesh et al., 2003).

En el modelo propuesto (ver tabla 3) se ha agregado una quinta categoría, la de la expectativa pedagógica. Esta categoría da cuenta de una de las variables que aparece con más fuerza en algunas de las entrevistas - básicamente en las correspondientes a los profesores experimentados y en menor medida en los entusiastas - que es el foco y la preocupación por el aprendizaje de los estudiantes.

$\mathrm{Al}$ integrar esta quinta categoría al modelo, los autores la vincularon con los enfoques de enseñanza de cada uno de los grupos encontrados: El grupo de profesores experimentados, que ha mostrado una inclinación hacia el enfoque centrado en el alumno, mostrará una alta relevancia del factor de expectativa pedagógica; el grupo de profesores cautelosos, que ha mostrado una inclinación hacia el enfoque centrado en el profesor, mostrará una baja relevancia hacia el factor de expectativa pedagógica. Por último, el grupo de profesores entusiastas oscila entre una actitud orientada al profesor y una orientada al alumno, con lo cual se considera que la relevancia del factor pedagógico en este caso es intermedia.

Volviendo a las preguntas de investigación con las cuales nos confrontamos a lo largo del trabajo:

¿En qué medida una situación de crisis puede impactar en la disposición del docente universitario a efectuar cambios pedagógicos en su práctica?

Dorfsman, M. \& Horenczyk, G. El cambio pedagógico en la docencia universitaria en los tiempos de Covid-19

Page 23 of 27 
¿Qué tipos de cambios pedagógicos se produjeron, si es que los hubo, en la práctica pedagógica de los docentes universitarios en tiempos de CRTS?

¿Cuáles son los factores favorecedores y obstaculizadores de dichos cambios?

Y derivado de estas: “¿De qué manera es dable caracterizar el cambio en la percepción de prácticas y concepciones pedagógicas en esta situación de emergencia?, es posible sostener que el cambio se puede producir como cambio formal o genuino, o bien un intermedio entre ambos. Cada uno de los grupos mencionados muestra una mayor o menor disposición a una de las modalidades de cambio pedagógico, y esta disposición está vinculada con el grado de aceptación de las tecnologías y con la concepción pedagógica del docente.

En suma, del trabajo es dable aprender que el cambio pedagógico mediado por tecnologías se vincula mayormente con dos factores; el nivel de alfabetización digital alcanzado por el docente, por un lado, y su concepción pedagógica de le enseñanza por el otro. El modelo UTAUT, enriquecido con el factor de expectativa pedagógica, puso de relieve una caracterización vinculada al primero de ellos y contribuyó a articular su predisposición a integrar tecnologías con la factibilidad de la realización de cambios pedagógicos genuinos en la enseñanza.

Para completar este trabajo, se propondrán posibles líneas de acción e investigación y las limitaciones del mismo.

\section{Futuras líneas de investigación}

El trabajo realizado ha analizado la primera etapa del Covid-19, que afectó a los sistemas universitarios educativos en Israel entre marzo de 2021 y hasta mayo, cuando se levantó la primera cuarentena.

La continuidad de la pandemia y sus consecuencias han llevado a las instituciones universitarias (en particular) y al sistema educativo (en general) a adaptarse a una situación de emergencia que ya se ha convertido en una especie de "rutina en emergencia".

Ante esta situación, en una segunda etapa de la investigación se sugiere preguntar sobre el efecto de esta situación de emergencia durante el primer semestre de 2020 (octubre), cuando, a diferencia del anterior, hubo tiempo suficiente para preparar sus clases en línea y además, los docentes ya contaban con experiencia previa en condiciones CRTS.

Entonces, en esta etapa será necesario indagar sobre la enseñanza que puede caracterizarse como "emergencia programada". ¿Cómo se prepararon los profesores para ello? ¿Qué tipo de prácticas se realizaron? ¿Qué aprendieron de la etapa anterior y cómo se puede caracterizar esta etapa?

$\mathrm{Al}$ entrevistar nuevamente a los docentes que participaron en la primera parte de esta investigación, también se procurará identificar cómo cada una de las categorías de la tipología presentada (experimentados, entusiastas y cautelosos) ha evolucionado durante esta segunda etapa. ¿Qué tipo de cambios se han producido en las prácticas y de qué manera las categorías identificadas en nuestra tipología pueden ser útiles para desarrollar nuevas líneas de formación pedagógica en el área de la docencia universitaria?

Dorfsman, M. \& Horenczyk, G. El cambio pedagógico en la docencia universitaria en los tiempos de Covid-19

Page 24 of 27 


\section{Limitaciones del estudio}

El presente estudio presenta limitaciones, algunas relacionadas con la metodología y otras con la conceptualización. Desde el punto de vista metodológico, es dable destacar una vez más el uso de métodos mixtos con énfasis cualitativo. Esto exige un arduo trabajo de combinación entre los dos métodos y la integración de los hallazgos obtenidos de ambos.

Otra limitación está relacionada con el muestreo. El estudio se basa en una muestra tomada de una sola universidad. Aunque la muestra tiende a ser representativa en relación con la población universitaria, no deja de ser una muestra que se reduce a una universidad en un país específico.

Desde un punto de vista conceptual, es importante tener en cuenta que los datos se recopilaron durante el primer mes de la crisis, el primer mes de la pandemia en Israel. No estaba claro qué estaba por suceder en el futuro mediato e inmediato. Es probable que esto haya afectado las percepciones y enfoques de los encuestados (en especial en la fase cualitativa de la investigación) y de los propios investigadores.

Entonces, al evaluar los resultados del estudio y aplicarlos a otros contextos y circunstancias, habrá que considerar las especificidades mencionadas en éste a fin poder justipreciar su relevancia para éstos.

\section{Agradecimientos}

Los autores agradecen la colaboración de las autoridades de la Universidad Hebrea por haber facilitado el acceso a los docentes de toda la casa de estudios; a todos los docentes que gentilmente accedieron a completar los cuestionarios suministrados y en forma especial a todos aquellos que gentilmente han accedido a participar de las entrevistas en profundidad, atendiendo a las normas éticas y de confidencialidad.

Presentación del artículo: 25 de marzo de 2021

Fecha de aprobación: 26 de junio de 2021

Fecha de publicación: 30 de septiembre de 2021

Dorfsman, M. \& Horenczyk, G. (2021). El cambio pedagógico en la docencia universitaria en los tiempos de Covid-19. RED. Revista Educación a Distancia, 21(67). http://dx.doi.org/10.6018/red.475151

\section{Financiación}

Esta investigación no recibe financiación y se realiza bajo el programa de colaboración internacional con la participación de los siguientes investigadores.

Initiators of the cross-cultural study: Horenczyk, G. and Dorfsman, M. (Hebrew University, Israel); Lion, C. (Universidad de Buenos Aires, Argentina); Göbel, K. (Universität Duisburg-Essen, Germany); Makarova, E. (Universität Basel, Switzerland); Birman, D. (Miami University, USA).

Dorfsman, M. \& Horenczyk, G. El cambio pedagógico en la docencia universitaria en los tiempos de Covid-19 


\section{Bibliografía}

Admiraal, W., Louws, M., Lockhorst, D., Paas, T., Buynsters, M., Cviko, A., . . Post, L. (2017). Teachers in school-based technology innovations: A typology of their beliefs on teaching and technology. Computers \& Education, 114, 57-68.

Alenezi, A. (2017). Obstacles for teachers to integrate technology with instruction. Education and Information Technologies, 22(4), 1797-1816.

Argibay, J. C. (2009). Muestra en investigación cuantitativa. Subjetividad y procesos cognitivos, 13-29.

Bebell, D., \& Kay, R. (2010). One to one computing: A summary of the quantitative results from the Berkshire wireless learning initiative. Journal of Technology, Learning, and Assessment, 9(2), n2.

Beetham, H., \& Sharpe, R. (2007). Rethinking pedagogy for a digital age: Designing and delivering e-learning: Routledge.

Bertaux, D. (1993). La perspectiva biográfica: validez metodológica y potencialidades. La historia oral: métodos y experiencias, 149-170.

Blau, I., \& Peled, Y. (2012). [Chais] Teachers' Openness to Change and Attitudes towards ICT: Comparison of Laptop per Teacher and Laptop per Student Programs. Interdisciplinary Journal of E-Learning and Learning Objects, 8(1), 73-82.

Braun, V., \& Clarke, V. (2012). Thematic analysis. In P. M. C. H. Cooper, D. L. Long, A. T. Panter, D. Rindskopf, \& K. J. Sher (Ed.), APA handbook of research methods in psychology (Vol. 2, Research designs: Quantitative, qualitative, neuropsychological, and biological pp. 57-71). Washington, DC.

Brown, K. L. (2003). From teacher-centered to learner-centered curriculum: Improving learning in diverse classrooms. Education, 124(1), 49-55.

Creswell, J. W., \& Clark, V. L. P. (2017). Designing and conducting mixed methods research. Thousand Oaks, CA: Sage Publications.

Davis, F. D. (1989). Technology Acceptance Model: TAM. Al-Suqri, MN, Al-Aufi, AS: Information Seeking Behavior and Technology Adoption, S, 205-219.

Dorfsman, M. (2018). El educador en línea: más allá de la digitalidad. RED. Revista de Educación a Distancia, 56.

Emaliana, I. (2017). Teacher-centered or student-centered learning approach to promote learning? Jurnal Sosial Humaniora (JSH), 10(2), 59-70.

Eshet-Alkalai, Y. (2004). Digital literacy: A conceptual framework for survival skills in the digital era. Journal of Educational Multimedia and Hypermedia, 13(1), 93-106.

Fenstermacher, G. D., \& Soltis, J. F. (2004). Approaches to teaching. New York: Teachers College Press.

Fishbein, M., \& Ajzen, I. (2011). Predicting and changing behavior: The reasoned action approach: Taylor \& Francis.

Fullan, M. (2002). El significado del cambio educativo: un cuarto de siglo de aprendizaje. Profesorado. Revista de currículum y formación de profesorado, 6(1-2), 1-14.

Graves, K. E., \& Bowers, A. J. (2018). Toward a Typology of Technology-Using Teachers in the" New Digital Divide": A Latent Class Analysis of the NCES Fast Response Survey System Teachers' Use of Educational Technology in US Public Schools, 2009 (FRSS 95). Teachers College Record, 120(8).

Hodges, C., Moore, S., Lockee, B., Trust, T., \& Bond, A. (2020). The difference between emergency remote teaching and online learning. Educause Review, 27, 1-12.

Dorfsman, M. \& Horenczyk, G. El cambio pedagógico en la docencia universitaria en los tiempos de Covid-19 
Kálmán, O., Tynjälä, P., \& Skaniakos, T. (2020). Patterns of university teachers' approaches to teaching, professional development and perceived departmental cultures. Teaching in Higher Education, 25(5), 595-614.

Kember, D., \& Kwan, K.-P. (2000). Lecturers' approaches to teaching and their relationship to conceptions of good teaching. Instructional Science, 28(5), 469-490.

Kreijns, K., Vermeulen, M., Kirschner, P. A., Buuren, H. v., \& Acker, F. V. (2013). Adopting the Integrative Model of Behaviour Prediction to explain teachers' willingness to use ICT: a perspective for research on teachers' ICT usage in pedagogical practices. Technology, Pedagogy and Education, 22(1), 55-71.

Kvale, S. (1996). Interviews: An introduction to qualitative research interviewing: Sage Publications, Inc.

Le Fevre, D. M. (2014). Barriers to implementing pedagogical change: The role of teachers' perceptions of risk. Teaching and Teacher Education, 38, 56-64.

McLoughlin, C., \& Lee, M. (2008). Mapping the digital terrain: New media and social software as catalysts for pedagogical change. Paper presented at the Ascilite Melbourne.

Murphy, M. P. (2020). COVID-19 and emergency eLearning: Consequences of the securitization of higher education for post-pandemic pedagogy. Contemporary Security Policy, 41(3), 1-14.

Navarrete, J. M. (2000). El muestreo en la investigación cualitativa. Investigaciones sociales, 4(5), 165-180.

Ortner, S. B. (1973). On Key Symbols. American Anthropologist, 75(5), 1338-1346.

Schreurs, J., \& Dumbraveanu, R. (2014). A shift from teacher centered to learner centered approach. International Journal of Engineering Pedagogy (iJEP), 4(3), 36-41.

Shkedi, A. (2003). Words of meaning: Qualitative research-theory and practice. Tel Aviv, Israel: Ramot.

Strauss, A., \& Corbin, J. (2007). Basics of qualitative research: Techniques and procedures for developing grounded theory: Sage Publications, Incorporated.

Tarling, I., \& Ng'ambi, D. (2016). Teachers pedagogical change framework: a diagnostic tool for changing teachers' uses of emerging technologies. British Journal of Educational Technology, 47(3), 554-572.

Terhart, E. (2013). Teacher resistance against school reform: Reflecting an inconvenient truth. School Leadership \& Management, 33(5), 486-500.

Venkatesh, V., \& Bala, H. (2008). Technology Acceptance Model 3 and a research agenda on interventions. Decision sciences, 39(2), 273-315.

Venkatesh, V., \& Davis, F. D. (2000). A theoretical extension of the Technology Acceptance Model: Four longitudinal field studies. Management Sciences, 46(2), 186204.

Venkatesh, V., Morris, M. G., Davis, G. B., \& Davis, F. D. (2003). User acceptance of information technology: Toward a unified view. MIS quarterly, 425-478.

Wood, E., Mueller, J., Willoughby, T., Specht, J., \& Deyoung, T. (2005). Teachers' perceptions: Barriers and supports to using technology in the classroom. Education, Communication \& Information, 5(2), 183-206.

Yan, Z. (2020). Unprecedented pandemic, unprecedented shift, and unprecedented opportunity. Human Behavior and Emerging Technologies, 2(2), 110-112.

Dorfsman, M. \& Horenczyk, G. El cambio pedagógico en la docencia universitaria en los tiempos de Covid-19 